\title{
Sea slugs, subliminal pictures, and vegetative state patients: boundaries of consciousness in classical conditioning
}

\author{
Tristan A. Bekinschtein ${ }^{1}$, Moos Peeters ${ }^{1}$, Diego Shalom ${ }^{2}$ and Mariano Sigman ${ }^{2}$ \\ Cognition and Brain Sciences Unit, Medical Research Council, Cambridge, UK \\ 2 Integrative Neuroscience Lab, Physics Department, University of Buenos Aires, Buenos Aires, Argentina
}

Edited by:

Mads Jensen, Aarhus University

Hospital, Denmark

\section{Reviewed by:}

Anil K. Seth, University of Sussex, UK Melanie Wilke, California Institute of Technology, USA

Giorgio Marchetti, www.

mind-consciousness-language.com

research net, Italy

*Correspondence:

Tristan A. Bekinschtein, Cognition and Brain Sciences Unit, Medical

Research Council, 15 Chaucer Road, CB2 TEF Cambridge, UK.

e-mail: tristan.bekinschtein@mrc-cbu. cam.ac.uk
Classical (trace) conditioning is a specific variant of associative learning in which a neutral stimulus leads to the subsequent prediction of an emotionally charged or noxious stimulus after a temporal gap. When conditioning is concurrent with a distraction task, only participants who can report the relationship (the contingency) between stimuli explicitly show associative learning. This suggests that consciousness is a prerequisite for trace conditioning. We review and question three main controversies concerning this view. Firstly, virtually all animals, even invertebrate sea slugs, show this type of learning; secondly, unconsciously perceived stimuli may elicit trace conditioning; and thirdly, some vegetative state patients show trace learning. We discuss and analyze these seemingly contradictory arguments to find the theoretical boundaries of consciousness in classical conditioning. We conclude that trace conditioning remains one of the best measures to test conscious processing in the absence of explicit reports.

\section{Keywords: Aplysia, subliminal, vegetative state, consciousness, learning, trace conditioning}

\section{INTRODUCTION}

Learning comes in many forms. Some forms, such as declarative memory, are explicit memories of objects, places, or events. Others forms, such as non-declarative memory, are implicit, like habits, skills, or priming, and do not require conscious awareness (Gilbert et al., 2001; Squire, 2004). While this has been an established taxonomy of learning, the boundaries are often vague and difficult to demarcate. For instance, in associative learning there is a fine-grained distinction between declarative associative learning, which is dependent on the conscious association of events, and non-declarative unconscious associative learning, which occurs without awareness of the link between the related meaningful events (Shanks, 1995).

In an associative learning task, when two stimuli are systematically presented in a temporal sequence, a new relationship between these two items is learned. In classical trace learning, a neutral conditioning stimulus (CS) precedes and, therefore, causes the subsequent prediction of an emotionally charged or noxious stimulus (unconditioned stimulus, US; Figure 1). During a differential eye-blink trace conditioning task, a specific tone (CS+) warns of a puff of air to the eye, whereas another tone (CS-) does not. The presence of an anticipatory eye-blink response, which is a conditioned response (CR) to the CS+ tone, is highly correlated with participants' verbal report of the relationship between the stimuli presented; this is known as the contingency (Dawson and Reardon, 1973; Clark and Squire, 1998). In contrast, during a delay conditioning task, for which there is no gap between the neutral stimulus (CS) and the puff of air to the eye, blink responses (CR) are elicited despite the lack of awareness of the contingency (Clark and Squire, 1998, 1999; Figure 1). Furthermore, trace, but not delay, conditioning is strongly influenced by an expectancy of the US (Clark et al., 2001).

Consequently, delay conditioning has been considered a hallmark of non-declarative learning and is systematically used as an associative learning task in vertebrates and invertebrates (Lavond et al., 1993). In contrast, human trace learning is dependent on conscious awareness of the contingency between stimuli (Christian and Thompson, 2003). Moreover it is this fact that has made human trace learning a potential "Turing-test" of consciousness (Koch, 2004).

Though this "turing-test" potential has been influential in behavioral neuroscience, three arguments could pose a serious challenge to this notion: (1) trace conditioning can be learnt by almost every animal, even invertebrate sea slugs (Glanzman, 1995); (2) trace conditioning can be elicited using subliminal stimuli (Esteves et al., 1994); and (3) clinically defined unconscious patients might learn trace conditioning (Bekinschtein et al., 2009). It is in addressing these three arguments that we will characterize the theoretical boundaries of consciousness of learning.

\section{IS CONDITIONING DIFFERENT BETWEEN SEA SLUGS AND HUMANS?}

The evolution of wings can be used as an analogy for the evolution of conditioning. During the evolution of animals on this planet, wings appeared at least three times from three different ancestors. The first appearance was probably 390-320 million years ago. Primitive wingless insects known as Bristletails used long antennae-like filaments at the ends of their bodies to glide down to tree trunks from forest canopies (Yanoviak et al., 2009). The second appearance, around 150 million years ago, involved the 


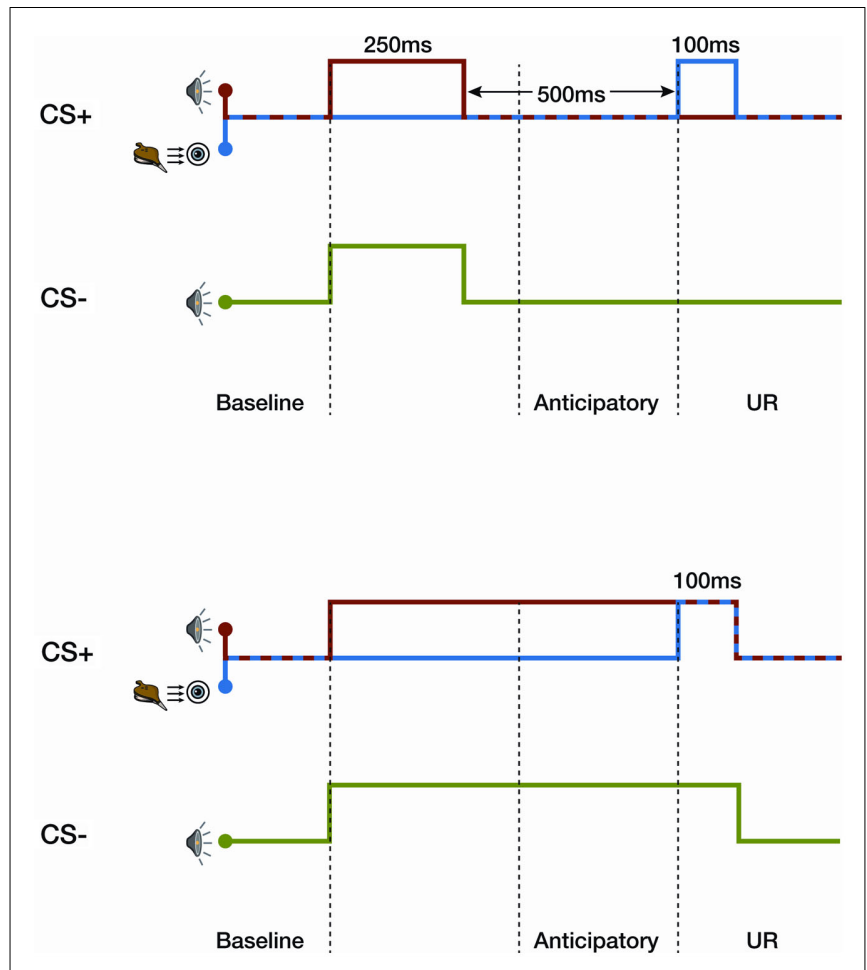

FIGURE 1 | Differential trace (upper graph) and delay conditioning (lower graph) for the eye-blink task. In trace conditioning there is a silent period between a tone and a puff of air to the eye. The CS+ tone is presented for $250 \mathrm{~ms}$, followed by a 500-ms silent gap, before a 100-ms puff of air to the eye (aversive stimulus). A second neutral stimulus tone, $\mathrm{CS}-$, is presented alone. In delay conditioning, the tone and puff of air co-occur. The tone lasts for $850 \mathrm{~ms}$, covering the silent gap and co-terminating with the puff of air.

development of wings on dinosaurs. Some dinosaurs started to evolve lighter skeletons; their wrists changed and feathers grew to form wings (Lewin, 1983; Sullivan et al., 2010). Though dinosaurs evolved wings, which gave birth to modern birds their capacity to fly has a different origin and ancestry than flying insects. The third appearance of wings developed in bats (Simmons et al., 2008). This is yet another origin of this feature. Though the three different wings serve a similar function, that is to fly, they were not derived from a common ancestor. Moreover, they are not controlled by the same machinery, and they do not obey the same rules.

Is the same true of conditioning? Has a conditioning mechanism appeared only once in evolution? While the well preserved molecular machinery may suggest this, findings at the systems level are disparate and may suggest a different conclusion (Barco et al., 2006). Molecular mechanisms underlying acquisition and consolidation of memory are in fact preserved in most species. However, learning relies on different network mechanisms in humans and sea slugs (Takehara et al., 2003). The plasticity of arrays of neurons and the ubiquitous evolutionary pressure of associative learning makes it extremely difficult to disentangle whether conditioning emerged only once during the course of evolution. It could be that conditioning emerged several times from several ancestral sources just as wings did. We are only left with the certainty that associative learning can be instantiated in simply a few neurons, as in sea slugs, or in millions of neurons, as in mammals.

Humans show the highest degree of behavioral flexibility among animals, probably through a flexible network that relies on a frontoparietal hub. This flexibility, however, comes at a cost that results in slow, limited computational capacity. Trace conditioning does not seem to escape from this rule (Zylberberg et al., 2010). Arbitrary associations in humans are instantiated in this routing system that appears to be intrinsically related to consciousness (Zylberberg et al., 2011). On the contrary, in the sea slug these temporary associations may rely on direct connections (Grol et al., 2006). This may also happen in humans with highly compatible sensory-motor relations which bypass the central routing system (Grol et al., 2006). Trace conditioning in sea slugs, where consciousness is not required, does not necessarily imply that consciousness does not play a role in human trace conditioning. Alone, it does not imply more than, for example, a bacteria in need of oxygen breathing under water makes implications about human respiration.

Trace conditioning it is also instantiated in neuroanatomically different systems. The sea slug Aplysia Californica only needs a few neurons to perform trace conditioning, while rabbits require hippocampal, frontal cortex, and cerebellar networks (Christian and Thompson, 2003). In contrast, rabbits seem to be more flexible than gastropods in their learning abilities, but not more than insects, i.e., fruit flies, (Heisenberg et al., 2001). In humans, as in rodents, trace conditioning is dependent of the hippocampus (James et al., 1987; Moyer et al., 1990; Clark and Squire, 1998) while delay conditioning can be elicited without hippocampi (Ivkovich and Stanton, 2001; Woodruff-Pak and Disterhoft, 2008). Delay conditioning seems to rely primarily on a functional cerebellum (McCormick and Thompson, 1984; Mauk and Thompson, 1987; Gerwig et al., 2007). Functional imaging studies have shown that the hippocampus is activated by both trace and delay conditioning, but it is significantly more activated by trace (Cheng et al., 2008). These studies of neuroanatomy and the function of associative conditioning may indicate declarative memory's high dependence on the hippocampi and neocortex, as opposed to nondeclarative memory's need for these structures, which is minimal (Eichenbaum and Cohen, 2000).

Further evidence of a causal link between awareness of the contingency and trace conditioning comes from human and nonprimate mammalian data. In humans the variability in trace conditioning responses and learning seems to be linked to attention (Lovibond and Shanks, 2002; Carter et al., 2003), suggesting a strong modulation by central cognitive processes. In healthy volunteers, increasing attentional load parametrically modulates the degree of trace learning (Carter et al., 2003). This elegant experiment used the classic $n$-back task to engage attentional resources and working memory. The engagement of these dramatically decreases the anticipatory responses to the US in trace conditioning. The effect was less prominent for delay conditioning. Interestingly, the interference paradigm used in humans to decrease conditioning has been replicated using mice (Han et al., 2003), demonstrating a similar functional frontotemporal network supporting successful trace conditioning in a non-primate 
mammalian species. In this study mice heard a tone and received a foot shock immediately after (delay conditioning) or, alternatively, the mice received a silent gap between tone and shock (trace conditioning). Two additional groups of mice experienced two lights flashing as interference for the tone-shock conditions. Learning was impaired by light interference in trace but not in delay conditioning. In mice the light flashing acted as "cognitive" interference, just as working memory load might cause interference in trace conditioning with humans. In both populations, i.e., in humans and mice, interference effects are found in trace but not delay conditioning. The fact that attention is a key component of conscious learning and also that trace conditioning is affected by awareness may indicate that trace conditioning is a type of conscious learning.

\section{IS TRACE CONDITIONING POSSIBLE WHEN STIMULI ARE UNCONSCIOUSLY PERCEIVED?}

There is general agreement that exposure to a contingency between conditioned stimulus (CS) and US will create an associative process called conditioning (Dickinson, 1980; Rescorla, 1988). The difference between human and non-human animal models is that the former can easily produce a verbal or motor report of the relationship between the CS and US. If humans can form an internal representation of the contingency and verbalize it or make a voluntary response, then this behavior is taken as evidence of conscious awareness (Lovibond and Shanks, 2002).

What if the CS is masked, or more generally, not accessible to verbal reports? Can a verbal report of the contingency still be present? This question is central to current theoretical discussions of the role of conscious processing in trace conditioning. Is conscious awareness of the stimuli needed in order for trace conditioning to occur? There are primarily two models that account for awareness in trace conditioning. The single-process model, asserts that a sole propositional learning process mediates expression CR and the expectancy of US (full network mapping; Lovibond and Shanks, 2002). The dual-process model, however, claims that these behavioral responses, both CR expression and US expectancy, are expressions of two independent learning processes (partial network mapping; Perruchet, 1985; Morris et al., 1999; Perruchet et al., 2006).

There is empirical evidence championing each model. Some studies find evidence in support of the single-process model (Daum et al., 1991, 1992; Manns et al., 2000a,b; Weike et al., 2007). While the series of experiments performed by Perruchet, Destrebecqz, Cleeremans, and colleagues (Destrebecqz and Cleeremans, 2001; Destrebecqz et al., 2005, 2010; Perruchet et al., 2006) and experiments performed by others (Ohman and Soares, 1993, 1994, 1998; Ohman et al., 1995; Weidemann et al., 2009) strongly support a two-way learning process.

The discussion is centered on the verbal reports of awareness of the contingencies (in Questionnaires, motor evaluation or subjective ratings) and the measures of CR. There is either complete agreement or disagreement between the two. This discrepancy raises two relevant methodological aspects about how to measure the awareness. Firstly, post-training questionnaires may elicit metacognitive process. Therefore the participant may verbally report the contingencies, not because they noticed them during the learning phase, but because they were forced to think about the contingencies after conditioning had finished. In this case it is difficult to know when conscious learning of the relationship between the stimuli occurred. It could have been during learning or it could have been when that participant was prompted about the relationship between stimuli. Secondly, if awareness is measured online during the experiment, as to avoid a post hoc metacognitive process, then participants may then take note of the contingencies of which they were previously unaware. This may lead to previously unaware participants becoming explicitly aware of the contingencies. The measurement of both verbal reports of contingencies and the CR is critical.

In a series of studies, Ohman et al. (1995) and Ohman and Soares $(1993,1994,1998)$ argue that conditioning of electrodermal responses to electric shocks can occur with masked, unconsciously perceived, stimuli. It has been found that unconsciously perceived stimuli only elicited a CR when fear-relevant stimuli, such as spiders and snakes (Ohman and Soares, 1993) or angry faces (Esteves et al., 1994), were presented as CS+. In support of Ohman's claims, one recent study showed that sensitivity to masking conditions was related to the CR of a masked CS but not an unmasked CS (Cornwell et al., 2007). In this study, sensitivity to the masked condition was a marker of unconscious processing, i.e., if participants were not aware of the masked item then this was taken to indicate that items were processed at an unconscious level. The depth of unconscious processing of the CS was linked to the intensity of the CR. Weak perception of the CS through masking may not elicit conscious recognition of it, but the CS may still be above an identification threshold. However, there are criticisms of these findings.

One criticism is that the measure used to assess perceptual awareness of the CS may not be sufficiently sensitive to identify participants with residual awareness of stimulus features (Pessoa, 2005; Graziano and Sigman, 2009). Additionally, some fear relevance effects in backward masking conditioning, as observed by Ohman, could be due to selective sensitization rather than unconscious associative processes. Some methodological concerns have also been raised concerning the extent to which participants were truly unaware of the stimuli (Lovibond and Shanks, 2002). Ohman replied to these criticisms thoroughly, using two main arguments. Firstly, not all verbal discriminative responses indicate awareness; discrimination of stimuli above chance levels does not necessarily imply conscious awareness (Merikle and Daneman, 2000; Wiens and Ohman, 2002). Secondly, criticisms have assumed that awareness is a conscious experience. Therefore a measure of awareness must involve a measure of a subjective state.

Another highly debated experiment was performed by Núñez and de Vicente (2004), they have also showed that CR can be elicited when masked words are paired with a mild shock, and that this response is, as it was in the studies by Ohman, related to the participants' detection threshold. However, the results of this study are somewhat difficult to interpret as a higher proportion of participants in the unconscious masked condition produced a CR than in the conscious group. This study evaluated masked words paired with electric shocks; they used either a detection threshold, i.e., Was the stimulus a word or a blank?, or an identification threshold, i.e., Was it word1, word 2 or not a 
word? When participants failed to detect a stimulus in the tachitoscope, half exhibited a CR above learning criterion (four out of eight). Yet, when participants were above detection threshold ("conscious") only 11\% (2 of 18) showed learning. There was a higher instance of conditioning, using a detection threshold, when stimuli were are presented unconsciously and when CR was measured using autonomic nervous system signals like skin conductance. On the contrary, when an identification threshold was applied, i.e., subjects had to differentiate between two words or two non-words, only $10 \%$ ( 1 of 10 participants) of participants in the unconscious condition exhibited CR, but 58\% (7 out of 12) of participants in the conscious group exhibited CR. These contradictory results point to two different learning systems, the unconscious system, which bypasses central processes and consciousness-related workspaces. It also possibly directly links the early visual system with the autonomic nervous system and the conscious associative system, where the activation of the frontoparietal cortices may influence the autonomous nervous system giving rise to a different signal and a different type of learning. It is indeed the case that the variability, speed, and regularity of the CR was higher in the conscious identification group as compared to the unconscious detection learners (Núñez and de Vicente, 2004).

There are, however, caveats to this study's design that must be considered when discussing the conclusions. Firstly, in the identification threshold condition, words were repeated and could have therefore been deprived of their meaning. When a word is repeated it becomes easy to rely on low level features, such as the letter array, to determine the word's identity without the need to access its meaning. The participants' decisions in this condition may not have been based on anything more than a surface features. Another methodological problem of this study is that the variability of the perceptual threshold was high and several subjects were therefore excluded leading to a small sample size. The final subgroups of learners were $2 / 18$ and $4 / 8$ for conscious and unconscious detection of a word/blank, respectively, and 7/12 and 1/10 subjects for conscious and unconscious identification of the word, respectively. The low number of participants complicates the statistical analysis and make conclusions difficult to extrapolate to other cases. Thirdly, there is another possible explanation of "unconscious conditioning". When an electric shock is used as the aversive stimulus, as opposed to a puff of air to the eye or a loud tone, it may induce a general increase in arousal. This may, change detection thresholds and act as a confounding factor, leading to difficulty in interpreting the results.

In short, there seems to be consistent evidence showing that trace conditioning can also be elicited by unconscious stimuli with a strong emotional content, which is not accessible for verbal report. This is reminiscent of the sea slug. There may be some specific residual forms of trace learning that can be mediated by the most likely candidates: unconscious processing and emotional stimuli. However, evidence of subliminal abstract, non-emotional stimuli eliciting trace conditioning is not conclusive. Careful and well-designed experiments are needed to robustly deliminate the boundaries of consciousness thresholds of stimuli in a given task. Their ability to elicit trace learning above and below this threshold should certainly be included in the agenda of highly relevant experiments in the next years.

\section{DO UNCONSCIOUS PATIENTS SHOW CONDITIONING?}

If trace conditioning is taken as an indirect test of awareness, then a clinically defined unconscious patient, i.e., those in a vegetative state (VS), that shows CRs (learning) should be considered conscious. Conversely, if a clinically defined unconscious patient shows anticipatory responses in trace conditioning, then this learning may not necessarily be indicative of conscious awareness (Bekinschtein et al., 2009). VS patients are considered to be unconscious. They lack any behavior consistent with conscious awareness, such as, they do not follow someone with their eyes or head across the room, and they do not gesticulate or react to signs, words or commands. However, we used a classic differential trace conditioning eye-blink paradigm in 13 VS patients to investigate whether these unconscious patients might show learning (Bekinschtein et al., 2009). Moreover, we found a subset of these VS patients did show learning (see Figure 2). Two VS could appropriately produce $\mathrm{CR}$ to $\mathrm{CS}+$ and not to $\mathrm{CS}-$, and another four patients showed non-discriminatory anticipatory responses, i.e., producing the $\mathrm{CR}$ to both stimuli types. Patients that demonstrated learning eventually recovered by regaining awareness, as opposed to those patients that did not show learning. It is our belief that the patients that showed learning were partially conscious at the time of testing, but were unable to produce overt voluntary responses. In a recent trace conditioning study, using aversive noises and pleasant fanfares measuring skin conductance response, Scott et al. (2011), showed that only participants attending to the stimuli and able to catch the rule of the experiment showed CRs. If this paradigm is applied in the same disorders of consciousness patients group as the trace eye-blink conditioning, it may show convergence in conscious-dependent learning, and helping to better define the learning-consciousness relationship.

It is crucial to consider alternate hypotheses related to trace conditioning and the limited capacity of consciousness. Limited capacity implies that to learn trace conditioning, progressive gain of awareness of the contingency is required by maintaining a free global workspace clear of other contents (Dehaene and Naccache, 2001). The use of this limited space for other events or processes may weaken the link that establishes the association between the CS and the US. This in turn would prevent the associative relationship from being established.

In our original study the control group for the unconscious patients were anesthetized participants. These sedated healthy participants showed no learning of the contingency, or CR, for eyeblink trace conditioning. The sedative (propofol) that anesthetized these participants ensured that they were indeed unconscious, and the levels of drug caused a significant decrease in absolute cerebral blood flow. In particular the propofol-related variations in the thalamic blood flow appeared to be linked to the midbrain reticular formation, thus suggests a close functional relationship between the two brain structures while unconscious (Fiset et al., 1999). These deeply sedated participants showed true unconsciousness through both diminished awareness and arousal (wakefulness). Moreover, the participants under low doses of propofol had a 


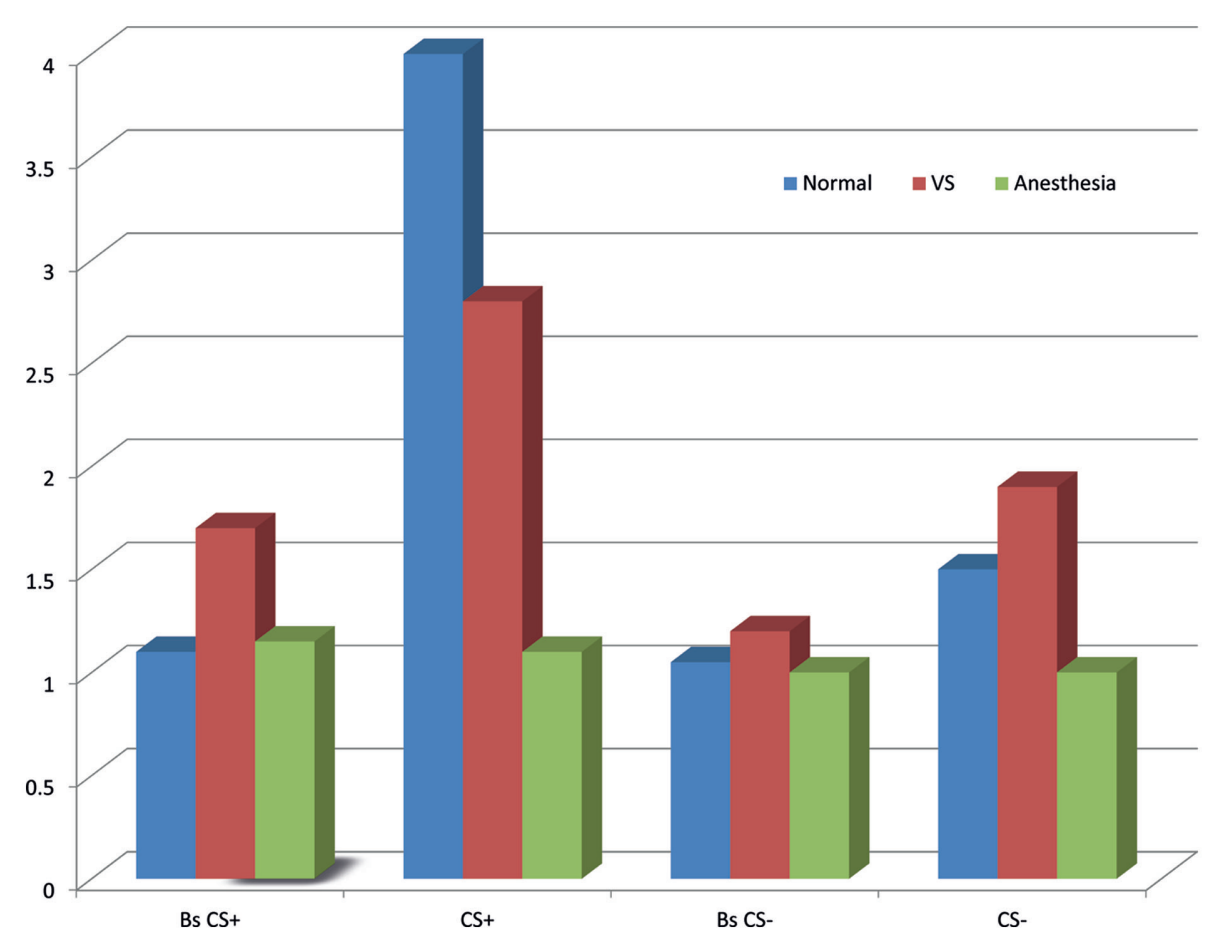

FIGURE 2 | Anticipatory learning in normal and anesthetized participants, and in vegetative state patients during trace differential conditioning. Bars show mean muscle activity for baseline of the $\mathrm{CS}+(\mathrm{Bs}$
$\mathrm{CS}+$ ), CS+ and baseline of the CS-(Bs CS-), CS-. Learning is in arbitrary units. Sedated subjects show no learning; vegetative state patients, as a group, show less muscle activity between CS+ and its baseline, and to CS- completely disorganized system for global integrated processing (Davis et al., 2007; Stamatakis et al., 2010). On the contrary, unconscious VS patients show dissociated wakefulness and awareness; they are regarded as being awake but not aware (Jennett and Plum, 1972). It could be that some VS patients retain partial functionality of the networks that support the acquisition of trace conditioning, but this is not enough to produce volitional movements.

\section{CONCLUSION}

Sea slugs can learn trace conditioning, but they do not show a form of learning that reveals the flexibility typically displayed in conscious forms of learning (Van den Bussche et al., 2008; Heinemann et al., 2009; see Dehaene and Changeux, 2011 for a review). Instead, these mollusks use the minimal numbers of systems necessary for successful associative learning. As far as we know sea slugs are not conscious, that is they are not conscious in the same way as humans are. Hence the mere observation of trace conditioning learning in this species does not provide evidence that trace conditioning is not an adequate signature of consciousness.

Quite the opposite is true; humans are extremely sensitive to context and are continuously interpreting all incoming stimuli in multiple ways. This capacity for over-interpretation may help in social interaction, goal-oriented behavior and possibly changes the way that trace conditioning is encoded and processed. The expression of trace learning in humans through a display of nonstereotyped representations of the contingency relies on an entirely distinct neural architecture. It also seems to rely on overt report in addition to CR.
Several studies have used masked or subliminally presented stimuli in order to make CS impossible to report (unconsciously perceived). This type of design has been used to explicitly determine whether conscious perception of the stimuli is necessary to achieve trace learning. Unfortunately, the results are inconclusive about whether trace learning, generally, is achievable through subliminal stimuli or under some specific circumstances. A robust conclusion concerning these studies involves the CS. When it is not neutral, but of negative valence, the masked stimuli seems to be processed up to the point of forming an association with the US, despite not being reported, detected, or discriminated.

The fact that clinically defined unconscious patients show trace learning suggest that they may have partial capacity for conscious awareness, as trace learning is dependant on some form of conscious awareness of the contingency. This result is further strengthened by data from sedated participants that show drug induced unconsciousness does not produce anticipatory responses above the baseline (Bekinschtein et al., 2009).

Trace conditioning remains very much linked to awareness of the contingency between CS and US. We believe it is a combination of the timing between stimuli and the variations on the CS that will allow us to better frame, over the next few years, the boundaries between this basic form of learning and conscious awareness.

The aforementioned analyses leads us to propose here three conditions which should be met in order for trace conditioning to be used as a test for conscious awareness: (1) a relatively stable, sustained attention to the stimuli, (2) a low central processing load to avoid interference and (3) a well defined stimuli, perceptually 
discernible and close in presentation time. If (1) is not met, then attention will deviate from the stimuli, and the creation of the association between CS and US will be disrupted. This would lead to sparse and inconsistent demonstration of the CR (Armony and Dolan, 2002). If (2) is not met then working memory capacity will be saturated by another task. Learning will then decrease, and as a result there will be low awareness of the contingencies. In this respect, trace conditioning seems to require the central resources of the attentional system in order for the association to be established, and this seems to be paired with awareness (Carter et al., 2003). If (3) is not met, then the stimuli will be ambiguous and the trace that is established will not be long-lasting or robust. This will lead to partial learning only and high variance in CR (Lovibond and Shanks, 2002; Sehlmeyer et al., 2009).

We would like to raise one final important point that has not yet been emphasized. It is of paramount importance if trace conditioning is to be used in practical terms. Clark and Squire (1998) established that in order to observe an anticipatory eye-blink response participants must be aware of the contingency at a level that is sufficient for a verbal report. The contrary, however, is not

\section{REFERENCES}

Armony, J. L., and Dolan, R. J. (2002). Modulation of spatial attention by fear-conditioned stimuli: an eventrelated fMRI study. Neuropsychologia 40, 817-826.

Barco, A., Bailey, C. H., and Kandel, E. R. (2006). Common molecular mechanisms in explicit and implicit memory. J. Neurochem. 97, 1520-1533.

Bekinschtein, T. A., Shalom, D. R., Forcato, C., Herrera, M., Coleman, M. R., Manes, F. F., and Sigman, M. (2009). Classical conditioning in the vegetative and minimally conscious state. Nat. Neurosci. 12, 1343-1351.

Carter, R. M., Hofstotter, C., Tsuchiya, N., and Koch, C. (2003). Working memory and fear conditioning. Proc. Natl. Acad. Sci. 100, 1399-1404.

Cheng, D. T., Disterhoft, J. F., Power, J. M., Ellis, D. A., and Desmond, J. E. (2008). Neural substrates underlying human delay and trace eyeblink conditioning. Proc. Natl. Acad. Sci. U.S.A. 105, 8108-8113.

Christian, K. M., and Thompson, R. F. (2003). Neural substrates of eyeblink conditioning: acquisition and retention. Learn. Mem. 10, 427-455.

Clark, R. E., Manns, J. R., and Squire, L. R. (2001). Trace and delay eyeblink conditioning: contrasting phenomena of declarative and nondeclarative memory. Psychol. Sci. 12, 304-308.

Clark, R. E., and Squire, L. R. (1998). Classical conditioning and brain systems: the role of awareness. Science 280, 77-81.

Clark, R. E., and Squire, L. R. (1999). Human eyeblink classical condition- ing: effects of manipulating awareness of the stimulus contingencies. Psychol. Sci. 10, 14-18.

Cornwell, B. R., Echiverri, A. M., and Grillon, C. (2007). Sensitivity to masked conditioned stimuli predicts conditioned response magnitude under masked conditions. Psychophysiology 44, 403-406.

Daum, I., Channon, S., and Gray, J. A. (1992). Classical conditioning after temporal lobe lesions in man: sparing of simple discrimination and extinction. Behav. Brain Res. 52, 159-165.

Daum, I., Channon, S., Polkey, C. E., and Gray, J. A. (1991). Classical conditioning after temporal lobe lesions in man: impairment in conditional discrimination. Behav. Neurosci. 105, 396-408.

Davis, M. H., Coleman, M. R., Absalom, A. R., Rodd, J. M., Johnsrude, I. S., Matta, B. F., Owen, A. M., and Menon, D. K. (2007). Dissociating speech perception and comprehension at reduced levels of awareness. Proc. Natl. Acad. Sci. U.S.A. 104, 16032-16037.

Dawson, M. E., and Reardon, P. (1973). Construct validity of recall and recognition postconditioning measures of awareness. J. Exp. Psychol. 98, 308-315.

Dehaene, S., and Changeux, J. P. (2011). Experimental and theoretical approaches to conscious processing. Neuron 70, 200-227.

Dehaene, S., and Naccache, L. (2001). Towards a cognitive neuroscience of consciousness: basic evidence and a workspace framework. Cognition 79, $1-37$.

true (both in the original Clark and Squire data and in our data making more than 200 participants combined): Some participants show verbal reports of the contingency, but do not show the CR of an anticipatory associative response. This simply may be because participants react in different ways to the anticipation of the air puff when they are aware that it is imminent. The most frequent reaction is a contraction of the muscle prior to the air puff, however, some participants may control this spontaneous response by relaxing and, inhibiting their blinks or by simply doing nothing. Hence, an improved statement is needed. While the absence of learning does not provide information concerning the participants' degree of awareness; the contrary is true. The presence of trace learning may be taken as evidence in favor of conscious awareness.

\section{ACKNOWLEDGMENTS}

Tristan A. Bekinschtein is supported by a Wellcome Trust Biomedical Research Career Development Fellowship. We thank Simon Strangeways for his help with the figures and Elisa Pye for critically reading the manuscript.

Destrebecqz, A., and Cleeremans, A. (2001). Can sequence learning be implicit? New evidence with the process dissociation procedure. Psychon. Bull. Rev. 8, 343-350.

Destrebecqz, A., Peigneux, P., Laureys, S., Degueldre, C., Del Fiore, G., Aerts, J., Luxen, A., Van Der Linden, M., Cleeremans, A., and Maquet, P. (2005). The neural correlates of implicit and explicit sequence learning: interacting networks revealed by the process dissociation procedure. Learn. Mem. 12, 480-490.

Destrebecqz, A., Perruchet, P., Cleeremans, A., Laureys, S., Maquet, P., and Peigneux, P. (2010). The influence of temporal factors on automatic priming and conscious expectancy in a simple reaction time task. Q. J. Exp. Psychol. 63, 291-309.

Dickinson, A. (1980). Contemporary Animal Learning Theory. Cambridge: Cambridge University Press.

Eichenbaum, H., and Cohen, N. J. (2000). From Conditioning to Conscious Recollection: Memory Systems of the Brain. New York: Oxford University Press.

Esteves, F., Parra, C., Dimberg, U. and Ohman, A. (1994). Nonconscious associative learning: Pavlovian conditioning of skin conductance responses to masked fearrelevant facial stimuli. Psychophysiology 31, 375-385.

Fiset, P., Paus, T., Daloze, T., Plourde G., Meuret, P., Bonhomme, V., HajjAli, N., Backman, S. B., and Evans, A. C. (1999). Brain mechanisms of propofol-induced loss of consciousness in humans: a positron emission tomographic study. J. Neurosci. 19, 5506-5513.

Gerwig, M., Kolb, F. P., and Timmann, D. (2007). The involvement of the human cerebellum in eyeblink conditioning. Cerebellum 6, 38-57.

Gilbert, C. D., Sigman, M., and Crist, R. E. (2001). The neural basis of perceptual learning. Neuron 31 , 681-697.

Glanzman, D. L. (1995). The cellular basis of classical conditioning in Aplysia californica - it's less simple than you think. Trends Neurosci. 18, 30-36.

Graziano, M., and Sigman, M. (2009). The spatial and temporal construction of confidence in the visual scene. PLoS ONE 4, 4909. doi:10.1371/journal.pone.0004909

Grol, M. J., de Lange, F. P., Verstraten, F. A., Passingham, R. E., and Toni, I. (2006). Cerebral changes during performance of overlearned arbitrary visuomotor associations. J. Neurosci. 26, 117-125.

Han, C. J., O'Tuathaigh, C. M., van Trigt, L., Quinn, J. J., Fanselow, M. S., Mongeau, R., Koch, C., and Anderson, D. J. (2003). Trace but not delay fear conditioning requires attention and the anterior cingulate cortex. Proc. Natl. Acad. Sci. U.S.A. 100, 13087-13092.

Heinemann, A., Kunde, W., and Kiesel, A. (2009). Context-specific primecongruency effects: on the role of conscious stimulus representations for cognitive control. Conscious. Cogn. 18, 966-976.

Heisenberg, M., Wolf, R., and Brembs, B. (2001). Flexibility in a single 
behavioral variable of Drosophila. Learn. Mem. 8, 1-10.

Ivkovich, D., and Stanton, M. E. (2001). Effects of early hippocampal lesions on trace, delay, and long-delay eyeblink conditioning in developing rats. Neurobiol. Learn. Mem. 76, 426-446.

James, G. O., Hardiman, M. J., and Yeo, C. H. (1987). Hippocampal lesions and trace conditioning in the rabbit. Behav. Brain Res. 23, 109-116.

Jennett, B., and Plum, F. (1972). Persistent vegetative state after brain damage: a syndrome in search of a name. Lancet 1, 734-737.

Koch, C. (2004). The Quest for Consciousness. Englewood, CO: Roberts and Publisher.

Lavond, D. G., Kim, J. J., and Thompson, R. F. (1993). Mammalian brain substrates of aversive classical conditioning. Annu. Rev. Psychol. 44, 317-342.

Lewin, R. (1983). "How did vertebrates take to the air?" Science 221, 38-39.

Lovibond, P. F., and Shanks, D. R. (2002). The role of awareness in Pavlovian conditioning: empirical evidence and theoretical implications. J. Exp. Psychol. Anim. Behav. Process. 28, 3-26.

Manns, J. R., Clark, R. E., and Squire, L. R. (2000a). Awareness predicts the magnitude of single-cue trace eyeblink conditioning. Hippocampus $10,181-186$.

Manns, J. R., Clark, R. E., and Squire, L. R. (2000b). Parallel acquisition of awareness and trace eyeblink. Learn. Mem. 7, 267-272.

Mauk, M. D., and Thompson, R. F. (1987). Retention of classically conditioned eyelid responses following acute decerebration. Brain Res. 403, 89-95.

McCormick, D. A., and Thompson, R. F. (1984). Neuronal responses of the rabbit cerebellum during acquisition and performance of a classically conditioned nictitating membrane-eyelid response. J. Neurosci. 4, 2811-2822.

Merikle, P. M., and Daneman, M. (2000). "Conscious vs. unconscious perception," in The New Cognitive Neurosciences, 2nd Edn, ed. M. S. Gazzaniga
(Cambridge, MA: MIT Press), 1295-1303.

Morris, J. S., Ohman, A., and Dolan, R. J. (1999). A subcortical pathway to the right amygdala mediating "unseen" fear. Proc. Natl. Acad. Sci. U.S.A. 96, 1680-1685.

Moyer, J. R., Deyo, R. A., and Disterhoft, J. F. (1990). Hippocampectomy disrupts associative learning of the trace conditioned eye-blink response in rabbits. Behav. Neurosci. 104, 243-252.

Núñez, J. P., and de Vicente, F. (2004). Unconscious learning. Conditioning to subliminal stimuli. Span. J. Psychol. 7, 13-28.

Ohman, A., Esteves, F., and Soares, J. J. F. (1995). Preparedness and preattentive associative learning: electrodermal conditioning to masked stimuli. J. Psychophysiol. 9, 99-108.

Ohman, A., and Soares, J. J. F. (1993). On the automatic nature of phobic fear: conditioned electrodermal responses to masked fear-relevant stimuli. J. Abnorm. Psychol. 102, 121-132.

Ohman, A., and Soares, J. J. F. (1994). "Unconscious anxiety": phobic responses to masked stimuli. J. Abnorm. Psychol. 103, 231-240.

Ohman, A., and Soares, J. J. F. (1998). Emotional conditioning to masked stimuli: expectancies for aversive outcomes following nonrecognized fear-relevant stimuli. J. Exp. Psychol. Gen. 127, 69-82.

Perruchet, P. (1985). A pitfall for the expectancy theory of human eyelid conditioning. Pavlov. J. Biol. Sci. 20, 163-170.

Perruchet, P., Cleeremans, A., and Destrebecqz, A. (2006). Dissociating the effects of automatic activation and explicit expectancy on reaction times in a simple associative learning task. J. Exp. Psychol. Learn. Mem. Cogn. 32, 955-965.

Pessoa, L. (2005). To what extent are emotional visual stimuli processed without attention and awareness? Curr. Opin. Neurobiol. 15, 188-196.

Rescorla, R. A. (1988). Pavlovian conditioning: it's not what you think it is. Am. Psychol. 43, 151-160.

Scott, R. B., Minati, L., Dienes, Z., Critchley, H. D., and Seth, A. K.
(2011). Detecting conscious awareness from involuntary autonomic responses. Conscious. Cogn. 20, 936-942.

Sehlmeyer, C., Schöning, S., Zwitserlood, P., Pfleiderer, B., Kircher, T., Arolt, V., and Konrad, C. (2009). Human fear conditioning and extinction in neuroimaging: a systematic review. PLoS One 4(6):e5865.

Shanks, D. R. (1995). The Psychology of Associative Learning. Oxford: Oxford University Press.

Simmons, N. B., Seymour, K. L., Habersetzer, J., and Gunnell, G. F. (2008). Primitive early Eocene bat from Wyoming and the evolution of flight and echolocation. Nature 451, 818-821.

Squire, L. R. (2004). Memory systems of the brain: a brief history and current perspective. Neurobiol. Learn. Mem. 82, 171-177.

Stamatakis, E. A., Adapa, R. M., Absalom, A. R., and Menon, D. K. (2010). Changes in resting neural connectivity during propofol sedation. PLoS ONE 5, e14224. doi:10.1371/journal.pone.0014224

Sullivan, C., Hone, D., Xu, X., and Zhang, F. (2010). The asymmetry of the carpal joint and the evolution of wing folding in maniraptoran theropod dinosaurs. Proc. Biol. Sci. 277, 2027-2033.

Takehara, K., Kawahara, S., and Kirino, Y. (2003). Time-dependent reorganization of the brain components underlying memory retention in trace eyeblink conditioning. J. Neurosci. 23, 9897-9905.

Van den Bussche, E., Segers, G., and Reynvoet, B. (2008). Conscious and unconscious proportion effects in masked priming. Conscious. Cogn 17, 1345-1358.

Weidemann, G., Tangen, J. M., Lovibond, P. F., and Mitchell, C. J. (2009). Is Perruchet's dissociation between eyeblink conditioned responding and outcome expectancy evidence for two learning systems? J. Exp. Psychol. Anim. Behav. Process. 35 169-176.

Weike, A. I., Schupp, H. T., and Hamm, A. O. (2007). Fear acquisition requires awareness in trace but not delay conditioning. Psychophysiology 44, 170-180.

Wiens, S., and Ohman, A. (2002). Unawareness is more than a chance event: comment on Lovibond and Shanks. J. Exp. Psychol. Anim. Behav. Process. 28, 27-31.

Woodruff-Pak, D. S., and Disterhoft, J. F. (2008). Where is the trace in trace conditioning? Trends Neurosci. 31, 105-112.

Yanoviak, S. P., Kaspari, M., and Dudley, R. (2009). Gliding hexapods and the origins of insect aerial behaviour. Biol. Lett. 5, 510-512.

Zylberberg, A., Dehaene, S., Roelfsema, P. R., and Sigman, M. (2011). The human Turing machine: a neural framework for mental programs. Trends Cogn. Sci. (Regul. Ed.) 15, 293-300.

Zylberberg, A., Fernández Slezak D., Roelfsema, P. R., Dehaene, S., and Sigman, M. (2010). The brain's router: a cortical network model of serial processing in the primate brain. PLoS Comput. Biol. 6, e1000765. doi:10.1371/journal.pcbi.1000765

Conflict of Interest Statement: The authors declare that the research was conducted in the absence of any commercial or financial relationships that could be construed as a potential conflict of interest.

Received: 30 September 2010; accepted: 31 October 2011; published online: 06 December 2011.

Citation: Bekinschtein TA, Peeters M, Shalom D and Sigman M (2011) Sea slugs, subliminal pictures, and vegetative state patients: boundaries of consciousness in classical conditioning. Front. Psychology 2:337. doi: 10.3389/fpsyg.2011.00337

This article was submitted to Frontiers in Consciousness Research, a specialty of Frontiers in Psychology.

Copyright (๑ 2011 Bekinschtein, Peeters, Shalom and Sigman. This is an openaccess article subject to a non-exclusive license between the authors and Frontiers Media SA, which permits use, distribution and reproduction in other forums, provided the original authors and source are credited and other Frontiers conditions are complied with. 